\title{
The domain architecture of JBP1 suggests synergy between J-base DNA binding and thymidine hydroxylase activity
}

\author{
Athanassios Adamopoulos", \#, Tatjana Heidebrecht", \#, Jeroen Roosendaal ${ }^{2}$, Wouter G. Touw ${ }^{1}$, \\ Isabelle Q. Phan ${ }^{3}$, Jos Beijnen ${ }^{2}$ and Anastassis Perrakis ${ }^{1, *}$
}

${ }^{1}$ Department of Biochemistry, Netherlands Cancer Institute, Plesmanlaan 121, 1066 CX Amsterdam, the Netherlands

${ }^{2}$ Department of Clinical Pharmacology, Netherlands Cancer Institute, Plesmanlaan 121, 1066 CX Amsterdam, the Netherlands

${ }^{3}$ Seattle Structural Genomics Center for Infectious Disease, Center for Global Infectious Disease Research, Seattle Children's Research Institute, 307 Westlake Ave N, Seattle, WA 98109

${ }^{\#}$ These authors contributed equally

*To whom correspondence should be addressed. Email: a.perrakis@nki.nl

The authors wish it to be known that, in their opinion, the first two authors should be regarded as joint First Authors ${ }^{1}$

${ }^{11}$ Lead contact: Anastassis Perrakis, a.perrakis@nki.nl 


\section{ABSTRACT}

JBP1 (J-DNA Binding Protein 1) contributes to biosynthesis and maintenance of base J ( $\beta$-D-glucosylhydroxymethyluracil), a modification of thymidine (T) confined to pathogenic protozoa. JBP1 has two known functional domains: an N-terminal thymidine hydroxylase (TH) homologous to the 5methylcytosine hydroxylase domain in TET proteins; and a J-DNA binding domain (JDBD) that resides in the middle of JBP1. Here we show that removing JDBD from JBP1 results in a soluble protein $(\triangle-$ JDBD) with the $\mathrm{N}$ - and $\mathrm{C}$-terminal regions tightly associated together in a well-ordered domain. This $\triangle$-JDBD domain retains thymidine hydroxylation activity in vitro, but displays a fifteen-fold lower apparent rate of hydroxylation compared to JBP1. Small Angle X-ray Scattering (SAXS) experiments on JBP1 and JDBD in the presence and absence of J-DNA, and on $\triangle$-JDBD, allowed us to generate low-resolution three-dimensional models. We conclude that $\triangle$-JDBD, and not the $\mathrm{N}$-terminal region of JBP1 alone, is a distinct folding unit. Our SAXS-based model supports the notion that binding of JDBD specifically to J-DNA can facilitate hydroxylation a T 12-14 bp downstream on the complementary strand of the J-recognition site. We postulate that insertion of the JDBD module in the $\triangle$-JDBD scaffold during evolution provided a mechanism to synergize between J recognition and T hydroxylation, ensuring inheritance of $\mathrm{J}$ in specific sequence patterns following DNA replication. 


\section{INTRODUCTION}

Base J ( $\beta$-D-glucosyl-hydroxymethyluracil) is a modified base that replaces $1 \%$ of thymine in kinetoplastid flagellates ${ }^{1}$, such as Trypanosoma, Leishmania and Crithidia. In Leishmania, $99 \%$ of J is located in telomeric repeats ${ }^{2-4}$ whereas $1 \%$ is in internal chromosomal positions (iJ) in positions where transcription starts ${ }^{5}$ or stops ${ }^{6}$.

Biosynthesis of $\mathrm{J}$ occurs in two-steps. First, the 5-methyl group of specific T's in the genome is Ohydroxylated, forming hydroxymethyluracil $(\mathrm{hmU})$. Second, a glucose molecule is transferred to $\mathrm{hmU}$, resulting in $\mathrm{J}^{7-9}$. The first step is catalyzed by both J-DNA Binding Proteins 1 and 2 (JBP1 and JBP2), that have a distinct thymidine hydroxylase domain (TH) in their $\mathrm{N}$-terminus ${ }^{10}$. The hydroxylation of $\mathrm{T}$ in oligonucleotides is dependent on the presence of $\mathrm{Fe}(\mathrm{II})$, 2-oxoglutarate $(2 \mathrm{OG})^{11}$. The discovery of the hydroxylation function of JBP1 has led to the discovery of the function of the mammalian TET family of enzymes that convert 5-methylcytosine $(5 \mathrm{mC})$ to 5 -hydroxymethylcytosine $(\mathrm{hmC})^{12}$, and have crucial roles in epigenetic regulation through modification of $5 \mathrm{mC}$ to $\mathrm{hmC}$. Several structures of TET and TET-like hydroxylase domains have been determined ${ }^{13-15}$, also in complex with $5 \mathrm{mC}$ and $\mathrm{hmC}$, providing significant insight in $5 \mathrm{mC}$ hydroxylation. However the limited sequence similarity between TET and JBP, underlined by large deletions and insertions in the TH fold, makes it impossible to deduce the structures of JBP1 and JBP2 from that of TET.

$J B P 1$, but not JBP2, specifically recognises base $J$ in $D N A^{16}$. This recognition is mediated by a short $\sim 150$ residue domain in the middle of JBP1, the J-DNA binding domain (JDBD) ${ }^{17}$. JDBD recognises JDNA with high affinity ( 10 nM) and remarkable specificity over normal DNA ( 10,000 fold). The structure of JDBD revealed a novel variant of the helix-turn-helix domain, with an unusually elongated turn between the recognition and the supporting helix. Importantly, we have shown that a single residue (Asp-525) in the recognition helix is almost entirely responsible for the specificity towards J-DNA, as the D525A mutation abrogated specificity towards J-DNA both in vitro and in vivo. JBP1 recognizes and binds J-DNA in two steps ${ }^{18}$. Pre-steady state kinetic data revealed that the initial binding of JBP1 to glucosylated DNA is very fast and followed by a second, much slower and concentration independent step. From this observation and small-angle neutron scattering (SANS) experiments, we inferred that JBP1 undergoes a conformational change upon binding to DNA, and postulated that this may allow the hydroxylase domain of JBP1 to make contact with the DNA and hydroxylate Ts in spatial proximity.

From what we know about the mechanism of J biosynthesis, it follows that the highly restricted distribution of J-base must be co-determined by the thymidine hydroxylases (JBP1 and JBP2) that catalyze the initial step in J synthesis. Using SMRT sequencing of DNA segments inserted into 
plasmids grown in Leishmania ${ }^{19}$, it has been shown that J modification usually occurs near G-rich sequences potentially capable of forming G-quadruplexes and at pairs of Ts on opposite DNA strands, separated by 12 nucleotides. That led Genest et $a l^{19}$ to propose a model in which JBP2 is responsible for initial J synthesis; then JBP1 binds to pre-existing $J$ and hydroxylates another $T$ that typically resides $13 \mathrm{bp}$ downstream (but not upstream) on the complementary DNA strand. This model provides a mechanism explaining how JBP1 can maintain existing J following DNA replication.

On the basis of these results we developed the conformational change model presented in ref. 18: We postulated that J-binding docks JBDB on duplex DNA, allowing the TH domain to come into contact with a $T$ that is preferably $13 \mathrm{bp}$ downstream the complementary strand and hydroxylate it. To further test this model, we set out to understand the domain organization of JBP1 and describe their three-dimensional organization alone and in relation with DNA.

Here, we present new deletion mutants and hydroxylase activity data of JBP1, that result to a new definition for the TH domain, and we also study the domain architecture of JBP1 by small angle X-ray scattering (SAXS). Developments in SAXS, namely the improved software and hardware, have established SAXS as a powerful tool for the analysis of molecular structures, also in the case of multidomain, flexible molecules ${ }^{20-24}$. Coupling size-exclusion chromatography to SAXS (SEC-SAXS) allows separating complexes from the constituent partners, degradation products, and eventual contaminants, allowing the determination of particle size and shape of macromolecules. Collecting and analysing SAXS data on different JBP1 deletion mutants and their complexes with J-DNA, allowed us to generate low-resolution three-dimensional models of JBP1 and its complex with DNA. Our data suggest synergy between the TH and JDBD domain (that is likely a recurrent fold in nature, not solely confined to JBP1 orthologues), is an evolutionary adaptation, crucial for replicating epigenomic information in kinetoplastids. 


\section{RESULTS}

\section{The $\mathrm{N}$ - and $\mathrm{C}$-terminal regions of JBP1 behave as a single folding unit}

Countless previous attempts to truncate JBP1 either C-terminally or $\mathrm{N}$-terminally (before or after the JDBD), to obtain the $\mathrm{N}$-terminal TH domain or a putative $\mathrm{C}$-terminal domain, had invariably failed to yield soluble protein in our hands. The crystal structure of JDBD showed that the $\mathrm{N}$ - and C-termini of this domain are in proximity (Figure 1A). As JDBD is in the middle of the JBP1 sequence (Figure 1B) we decided to test the possibility that the JDBD is an insertion domain into a "TH domain" fold that spans the rest of the JBP1 sequence. To validate this hypothesis, we replaced the JDBD domain with a connecting linker. Remarkably, the resulting protein (JBP1 $\left.1^{1-382 / 561-882}, \triangle-J D B D\right)$ expressed well in soluble form, and could be purified in good amounts (Figure 1C). Intrigued by that, we wanted to examine if the $\mathrm{N}$-terminal (1-382) and the C-terminal (561-882) regions form a single folding unit, or behave as separate domains. We therefore introduced a $3 \mathrm{C}$ protease cleavage site in the connecting linker between the two halves of $\triangle$-JDBD (Figure-1B). Overexpression of this $\triangle$-JDBD-3C construct also yielded soluble protein (Figure $1 \mathrm{C}$ ). Incubation with $3 \mathrm{C}$ protease over a period of $13-36 \mathrm{hr}$ resulted in the protein chain to be cleaved in two, and these bands could be observed on an SDSPAGE (Figure 1C). However, when we run the cleaved protein on a size exclusion chromatography (SEC) column in the absence of detergent, the elution profile showed a single symmetric peak (Figure 1D) of approximately the same molecular weight as $\triangle$-JDBD, while the SDS-PAGE analysis of the eluted fractions confirmed that this peak has both bands present. This strongly suggests that the two polypeptides behave as one protein, indicating a strong interaction between the two termini. This indicates that both termini should be part of the same folding unit, which is the folding unit necessary to provide a functional catalytic site containing the TH activity. This experiment led us to revisit our previous view of JBP1, with an N-terminal TH domain, followed by the JDBD domain and a mysterious C-terminal region. We now hypothesized, that two folding units compose JBP1: the $\triangle$ JDBD and the JDBD that is "inserted" in the $\triangle$-DJBD folding scaffold.

\section{JBP1, JDBD, $\triangle$-JDBD are all well-folded globular domains in solution}

To further characterize $\triangle$-DJBD in solution, we performed Small Angle X-ray Scattering experiments on JBP1, JDBD, and $\triangle$-JDBD. All samples were injected in a size exclusion chromatography column (SEC), and the SAXS profile (as well and the absorption spectrum) were analysed under flow. All samples eluted as single peaks from the SEC and the SAXS curves from the absorption peak region were averaged to obtain a scattering curve for each component (Figure 2A and Supplemental Table 1). Standard analysis tools from the ATSAS ${ }^{32}$ and SCÅTTER ${ }^{20}$ suites were used to obtain modelindependent parameters (Table 1). To confirm that all samples were properly folded we performed a 
dimensionless Kratky plot analysis (Figure 2B). This plot, allows to compare the shape of particles independent of their size, and shows that both JBP1 and $\triangle$-JDBD have a similar profile with a maximum close to 1.104 , characteristic of compacted and folded molecules. The JDBD peak is shifted slightly right and upward, suggesting that JDBD is less globular but compacted (which agrees with the crystal structure shape). The pair distribution function (Figure $2 \mathrm{C}$ ) is compatible with this analysis, and together they confirm our interpretation of the biochemical experiments, suggesting that $\Delta$ JDBD is a stable single domain.

\section{$\triangle$-JDBD is a catalytically active domain that has thymidine hydroxylase activity}

We first established a mass spectrometry-based assay to measure TH activity of JBP1 in vitro. We used purified proteins with a 14-mer oligonucleotide in conditions similar to those reported in ${ }^{46}$ to convert $\mathrm{T}$ to $\mathrm{hmU}$, which was measured by quantitative liquid chromatography mass spectrometry, after converting the oligonucleotide to nucleosides (see Methods for details). This activity was fully dependent on the presence of the co-factor 2-oxoglutarate, and showed a modest but appreciable dependency to $\mathrm{Fe}^{+2}$, ascorbic acid as a reducing agent, and to buffer degassing (Supplementary Figure 1). We then monitored the rate of catalysis over time for both JBP1 and $\triangle$-JDBD. $\triangle$-JDBD was clearly active, but showed a catalytic rate of about 17-times lower than wild type JBP1 (Figure 3).

These experiments clearly establish that $\triangle$-JDBD is a well-folded active TH domain, which is not disrupted by splicing out the JDBD domain. We thus decided to then characterise the relative domain architecture between $\triangle$-JDBD and JDBD in the context of the JBP1 protein.

\section{Modelling of JBP1 as a two-domain ( $\triangle$-JDBD and JDBD) molecule shows flexibility for JDBD}

First, we compared further JBP1 to $\triangle$-JDBD by examining the Porod-Debye plot (Figure 4A). The presence of a plateau for $\triangle$-JDBD indicates that it forms a distinct particle with sharp scattering contrast. This feature is not present in JBP1, indicating a more diffuse scattering contrast for the full length JBP1. The same is observed calculating the packing densities for both $\triangle$-JDBD and JBP1: $\triangle$ JDBD has a packing density of $0.91 \mathrm{~g} \mathrm{~cm}^{-3}$ compared with $0.79 \mathrm{~g} \mathrm{~cm}^{-3}$ of JBP1. The observed diffusion in scattering contrast and the reduced packing density in the wild type, full-length, JBP1 compared to $\triangle$-JBP1 suggest that the JDBD domain is flexible with respect to the $\triangle$-JDBD scaffold.

To validate this hypothesis we decided to create $a b$ initio three-dimensional models based on the SAXS data. As we have SAXS data for $\triangle$-JDBD and JDBD alone, as well as for both of them together (JBP1), we decided it is more appropriate to model them with the procedures developed for macromolecular complexes and multi-domain proteins ${ }^{32,35}$. The program MONSA from the ATSAS suite $^{32}$ seeks to identify so called "multi-phase" models (each "phase" being a rigid domain) that fit simultaneously the scattering data describing each phase (domain) separately, and their complex. 
We defined two phases, $\triangle$-JDBD and JDBD, which make up a "complex", JBP1. Twenty models were created by MONSA to fit the three available scattering data sets. Details for this and subsequent MONSA modelling runs are in Table 2.

An examination of the individual models showed that the JDBD domain adopts multiple conformations with respect to the $\triangle$-JDBD scaffold. Clustering analysis with the program DAMCLUST from the ATSAS suite ${ }^{32}$ identified five clusters (Figure 4B), all of which have JDBD located on the same end of the elongated $\triangle$-JDBD scaffold, but in various positions around the long axis of the $\Delta$ JDBD domain (Figure $4 C$ ). If $\triangle$-JDBD is viewed as an ellipsoid, the JDBD is consistently positioned towards one half of the ellipsoid, but adopts multiple conformations around the long axis of the ellipsoid. This analysis is compatible with the model-independent analysis of the SAXS data and strengthens our previous hypothesis, that the JDBD domain is flexible with respect to the $\triangle$-JDBD scaffold.

\section{Binding of JBP1 to J-DNA leads to reduced flexibility of the JDBD domain}

We have previously shown that JBP1 and J-DNA complex formation is accompanied by a conformational change ${ }^{18}$. Our new data allow us to formulate the hypothesis that this conformational change might be the ordering of the JDBD: when JBP1 binds to DNA, JDBD might adopt a more defined conformation with respect to the $\triangle$-JBP1 scaffold. To test this hypothesis, we used the SEC-SAXS data on JBP1 in complex with 23-mer J-DNA (J-23-DNA). SEC-SAXS data were first collected for J-23-DNA, which had the expected parameters for an elongated molecule (Table 1 and Supplemental Table 1). The complex between J-23-DNA and JBP1 eluted from the SAXS column as a single peak, and was confirmed by the $280 / 260 \mathrm{~nm}$ absorption ratio; the averaged SAXS profile from the elution peak are shown in Figure 4A; model-independent parameters are in Table 1 and SEC details in Supplemental Table 1.

While visual inspection of the scattering intensity for JBP1 alone and the complex suggests that they are very similar (Figure 5A), plotting the intensity ratio of the two datasets shows that the molecular form factors for the two datasets have prominent differences, as we observe strong features throughout the curve (Figure $5 \mathrm{~B}$ ). Analysis of the data also shows that an increase in $\mathrm{R}_{\mathrm{g}}$ was accompanied by an increase in $D_{\max }$, suggesting that J-DNA binds away from the centre of mass (Table 1). The volume-of-correlation, $\mathrm{V}_{\mathrm{c}}$, is higher in the J-DNA-bound state, similar to the Porod volume that also increases by $14,000 \AA^{3}$ in the presence of J-23-DNA. Finally, examination of the dimensionless Kratky plot reveals a shift away from the Guinier-Kratky point (1.104) indicating that upon J-DNA binding, JBP1 has a more elongated shape (Figure $5 \mathrm{C}$ ). All these data establish that the 
complex of JBP1 and J-23-DNA is formed, and that the J-23-DNA binds away from the JBP1 centre-ofmass resulting in a more elongated particle.

To visualize the relative position of J-DNA in the complex, we again used the program MONSA. We defined two phases, J-23-DNA and JBP1, and calculated 20 models consistent with the scattering data for JBP1, J-23-DNA and the JBP1:J-23-DNA complex. Cluster analysis with DAMCLUST, resulted in four major clusters (Figure 6). In all clusters J-DNA is located towards one end of the JBP1 ellipsoid, away from its centre of mass, compatible with the positioning of the JDBD domain. In contrast with the two-body modelling of JDBD and $\triangle-J B P 1$, the clusters are rather similar, suggesting that J-23-DNA binds in similar conformations. As previous SAXS data on the complex between JDBD and J-DNA suggested that this is a rigid complex without conformational flexibility ${ }^{25}$, this postulates that this JDBD: J-23-DNA rigid complex is now in one conformation with respect to $\triangle$-JDBD. In other words, this analysis is compatible with the hypothesis that the JDBD gets ordered upon J-DNA binding. To confirm these finding, we repeated the procedure with 15mer J-DNA (J-15-DNA); the results (Table 1 and Supplementary Figure 2) lead to the same conclusions.

We then proceeded to test our hypothesis for the ordering of the JDBD upon complex formation, using a different modelling approach. For this, we treated the JDBD complex with J-DNA as a rigid body ${ }^{25}$ and defined two different phases: $\triangle$-JBP1 and the JDBD:J-23-DNA complex. We again used MONSA for calculating 20 models consistent with the scattering data for $\triangle$-JBP1, and the JDBD:J-23DNA and JBP1:J-23-DNA complexes. Cluster analysis with DAMCLUST resulted in three clusters (Supplementary Figure 3). Consistent with the previous analysis, these clusters are fairly similar, confirming the reduced flexibility of the complex of JBP1 with J:DNA, and again show the DNA and JDBD located towards one end of the complex.

As we have an atomic model for the JDBD:J-23-DNA complex ${ }^{25}$, we were able to place it in the respective dummy atom model using SUPCOMB ${ }^{39}$. Thus, we created a pseudo-atomic hybrid model, where the $\triangle$-JDBD (for which we do not have an atomic model) is shown as the dummy atom model, and JDBD and J-23-DNA are all-atom models. Using $\mathrm{CRYSOL}^{42}$ we evaluated the fit of both the dummy atom reconstruction and the pseudo-atomic hybrid model against the SAXS curve for JBP1:J23-DNA. The dummy atom reconstructions for the two most populated clusters, with ten and six members respectively, show the best fit to the experimental data ( $\chi=2.32$ and $\chi=1.68$ respectively). However, the pseudo-atomic hybrid model corresponding to the most populous cluster shows a considerably better fit to the experimental data $(\chi=6.87)$ compared to the second cluster $(\chi=38.03)$. Thus, we consider this model as the most likely interpretation of our experimental data for the complex of full length JBP1 with J-DNA (Figure 7). 
This pseudo-atomic model now shows the position of the J-base and the most likely orientation of the DNA. Interestingly, in this model, the T base 13bp away from $J$ in the complementary strand gets in contact with the $\triangle$-JDBD domain that contains the TH activity.

\section{DISCUSSION}

The discovery that JBP1 has an N-terminal TH domain sequence signature, which likely functions as a thymidine hydroxylase ${ }^{10}$, was an important finding for the field of J biosynthesis. Perhaps more remarkably, this sparked a revolution for the methylcytosine to hydroxymethylcytosine field ${ }^{12}$. Together with subsequent experimental proof of the TH activity hypothesis ${ }^{11}$, these findings established the notion that JBP1 consists of an N-terminal TH domain, followed by a J-DNA binding domain $^{25}$, and a C-terminal sequence which received little attention. Here, we show that the JDBD should be seen as an insertion domain within a single $\mathrm{TH}$ domain that spans the $\mathrm{N}$-terminal and $\mathrm{C}$ terminal sequence regions of JBP1, and behaves as a single folding unit in solution; $\triangle$-JDBD. We note that a JBP1 construct spanning residues 1-451 which has been previously reported to have hydroxylase activity ${ }^{47}$, as well as numerous other constructs of the $\mathrm{N}$-terminal from a variety of species, does not yield soluble protein in our hands. The sole exception to that rule has been the $\Delta$ JDBD domain. Remarkably, this new folding unit is functional as a thymidine hydroxylase, in a new enzymatic activity assay that we developed, having an apparent catalytic rate about 17 times slower compared to full-length JBP1. We suggest that this lower rate is explained by the inability of $\triangle$-JDBD to bind to DNA, bringing it in proximity to its T base substrates.

We have previously shown that binding of JBP1 to J-DNA is followed by a conformational change of $J B P 1^{18}$. Here, we extend this model, providing data that this conformational change represents a transition of JDBD: while JDBD is flexible with respect to the $\triangle$-JDBD scaffold in the absence of J-DNA, it gets ordered in the presence of J-DNA. This is in agreement with our previous observation from SANS data ${ }^{18}$ that the protein apparent radius of gyration $\left(R_{\mathrm{g}}\right)$ is reduced upon complexation with JDNA. This is consistent with JDBD sampling a more defined conformation space and reducing the apparent size of the protein particle.

The complex between JDBD and J-DNA is a well-defined rigid structure, as shown by our previous structural analysis ${ }^{44}$ and current data. Our current analysis of $\triangle$-JDBD and the JBP1 complex with JDNA shows these to be also fairly rigid. These allowed us to propose a pseudo-atomic hybrid model, showing the orientation of J-DNA with respect to the $\triangle$-JDBD domain that contains the TH catalytic activity (Figure 7). In that model, the DNA gets in contact with the $\triangle$-JDBD. The J-23-DNA sequence we used for these experiments contains both the $J$ that is recognized by the JDBD, but also a complementary strand sequence that is amenable to hydroxylation. Interestingly, in our most 
probable model, the $\mathrm{T}$ that lies $13 \mathrm{bp}$ downstream in the complementary strand comes in close contact with the $\triangle$-JDBD domain that has the TH activity. Thus, our structural analysis supports the hypothesis that the JDBD domain of JBP1 binds to J, and the $\triangle$-JDBD domain then undergoes a conformational change allowing it to reach and hydroxylate a T $13 \mathrm{bp}$ on the complementary strand. In this way JBP1 is able to maintain existing J following DNA replication (Figure 8).

From an evolutionary perspective, it is reasonable to presume that the thymidine hydroxylation activity to make hydroxymethyluracil precedes the glucosylation step to make J. We hypothesize that the last evolutionary step was the acquisition of J-binding activity, to guide the TH activity to areas of pre-existing $\mathrm{J}$ to replicate that epigenetic marker in kinetoplastids. As we show here, JDBD has likely been acquired by JBP1 through an insertion event that did not disturb the TH scaffold. Based on these observations, one would expect to find JDBD homologues - with or without specificity for J-DNA binding - in additional proteins. As sequence searches in public databases do not reveal clear homologues of the JDBD outside the context of JBP1 orthologues, we performed structural similarity searches using $\mathrm{DALI}^{43}$ (see Methods for details). These searches revealed two new structural homologues additional to $\operatorname{MogR}^{48}$, which we have previously described ${ }^{25}$. The closest structural homologue of JDBD is AcrF3, belonging to a family of proteins produced by bacteriophages to inactivate the CRISPR-Cas bacterial immune system ${ }^{45}$; the other homologue is a CTerminal helical domain (CHCT) of the chromatin remodelling protein $\mathrm{CHD} 1^{49}$. While JDBD, MogR, and CHCT clearly have a conserved positive patch for interaction with DNA (Figure 9), this patch is absent in AcrF3. Interestingly, anti-CRISPR (Acr) proteins bind the Cas complexes blocking recognition of double-stranded DNA substrates ${ }^{50,51}$ : speculating that the ancestry of Acr proteins is related to the JDBD, MogR and CHCT DNA recognition domains, this might present an extreme example of repurposing a DNA-recognition structural domain for preventing DNA-recognition, or vice versa. That data suggests that the JDBD DNA-recognition scaffold might be considerably more widespread and not confined to specific J recognition; as complete sequences of protozoan species will continue to be fully assembled, more JDBD-like domains will likely be identified, inside or outside the J-biosynthesis pathway.

\section{Supplementary Data are at the end of the manuscript}




\section{ACKNOWLEDGEMENT}

We thank Piet Borst for extensive discussions and suggestions to improve the text of this manuscript and Piet Borst and Henri van Luenen for helping to establish the JBP1 activity assays.

\section{FUNDING}

This work was supported by the Nederlandse Organisatie voor Wetenschappelijk Onderzoek [grant number 714.014.002]. Work from the SSGCID is supported by Federal funds from the National Institute of Allergy and Infectious Diseases, National Institutes of Health, Department of Health and Human Services, under Contract No.: HHSN272201700059C

\section{REFERENCES}

1. Gommers-Ampt, J. H. et al. beta-D-glucosyl-hydroxymethyluracil: A novel modified base present in the DNA of the parasitic protozoan T. brucei. Cell 75, 1129-1136 (1993).

2. van Leeuwen, F. et al. Localization of the modified base $\mathrm{J}$ in telomeric VSG gene expression sites of Trypanosoma brucei. Genes Dev 11, 3232-3241 (1997).

3. van Leeuwen, F. et al. The telomeric GGGTTA repeats of Trypanosoma brucei contain the hypermodified base J in both strands. Nucleic Acids Res 24, 2476-2482 (1996).

4. Genest, P.-A., Riet, ter, B., Cijsouw, T., van Luenen, H. G. A. M. \& Borst, P. Telomeric localization of the modified DNA base $\mathrm{J}$ in the genome of the protozoan parasite Leishmania. Nucleic Acids Res 35, 2116-2124 (2007).

5. Cliffe, L. J., Siegel, T. N., Marshall, M., Cross, G. A. M. \& Sabatini, R. Two thymidine hydroxylases differentially regulate the formation of glucosylated DNA at regions flanking polymerase II polycistronic transcription units throughout the genome of Trypanosoma brucei. Nucleic Acids Res 38, 3923-3935 (2010).

6. van Luenen, H. et al. Glucosylated Hydroxymethyluracil, DNA Base J, Prevents Transcriptional Readthrough in Leishmania. Cell 150, 909-921 (2012).

7. Bullard, W., Lopes da Rosa-Spiegler, J., Liu, S., Wang, Y. \& Sabatini, R. Identification of the glucosyltransferase that converts hydroxymethyluracil to base $\mathrm{J}$ in the trypanosomatid genome. Journal of Biological Chemistry 289, 20273-20282 (2014).

8. Iyer, L. M., Zhang, D., Maxwell Burroughs, A. \& Aravind, L. Computational identification of novel biochemical systems involved in oxidation, glycosylation and other complex modifications of bases in DNA. Nucleic Acids Res 41, 7635-7655 (2013).

9. Sekar, A., Merritt, C., Baugh, L., Stuart, K. \& Myler, P. J. Tb927.10.6900 encodes the glucosyltransferase involved in synthesis of base $\mathrm{J}$ in Trypanosoma brucei. Mol Biochem Parasitol 196, 9-11 (2014).

10. Yu, Z. et al. The protein that binds to DNA base $J$ in trypanosomatids has features of a thymidine hydroxylase. Nucleic Acids Res 35, 2107-2115 (2007).

11. Cliffe, L. J. et al. JBP1 and JBP2 Proteins Are Fe2+/2-Oxoglutarate-dependent Dioxygenases Regulating Hydroxylation of Thymidine Residues in Trypanosome DNA. Journal of Biological Chemistry 287, 19886-19895 (2012).

12. Tahiliani, M. et al. Conversion of 5-methylcytosine to 5-hydroxymethylcytosine in mammalian DNA by MLL partner TET1. Science 324, 930-935 (2009).

13. Hu, L. et al. Crystal Structure of TET2-DNA Complex: Insight into TET-Mediated 5mC Oxidation. Cell 155, 1545-1555 (2013).

14. Hashimoto, H. et al. Structure of a Naegleria Tet-like dioxygenase in complex with 5- 
methylcytosine DNA. Nature - (2013). doi:10.1038/nature12905

15. Hu, L. et al. Structural insight into substrate preference for TET-mediated oxidation. Nature 527, 118-122 (2015).

16. Cross, M. et al. The modified base $J$ is the target for a novel DNA-binding protein in kinetoplastid protozoans. EMBO J 18, 6573-6581 (1999).

17. Heidebrecht, T. et al. The structural basis for recognition of base J containing DNA by a novel DNA binding domain in JBP1. Nucleic Acids Res 39, 5715-5728 (2011).

18. Heidebrecht, T. et al. Binding of the J-Binding Protein to DNA Containing Glucosylated hmU (Base J) or 5-hmC: Evidence for a Rapid Conformational Change upon DNA Binding. J Am Chem Soc 120731143221009 (2012). doi:10.1021/ja303423t

19. Genest, P.-A. et al. Defining the sequence requirements for the positioning of base J in DNA using SMRT sequencing. Nucleic Acids Res 43, 2102-2115 (2015).

20. Rambo, R. P. \& Tainer, J. A. Bridging the solution divide: comprehensive structural analyses of dynamic RNA, DNA, and protein assemblies by small-angle X-ray scattering. Curr Opin Struct Biol 20, 128-137 (2010).

21. Petoukhov, M. V. \& Svergun, D. I. Analysis of X-ray and neutron scattering from biomacromolecular solutions. Curr Opin Struct Biol 17, 562-571 (2007).

22. Bizien, T. et al. A Brief Survey of State-of-the-Art BioSAXS. Protein Pept. Lett. 23, 217-231 (2016).

23. Vestergaard, B. Analysis of biostructural changes, dynamics, and interactions - Small-angle Xray scattering to the rescue. Arch. Biochem. Biophys. 602, 69-79 (2016).

24. Schindler, C. E. M., de Vries, S. J., Sasse, A. \& Zacharias, M. SAXS Data Alone can Generate High-Quality Models of Protein-Protein Complexes. Structure 24, 1387-1397 (2016).

25. Heidebrecht, T. et al. The structural basis for recognition of base J containing DNA by a novel DNA binding domain in JBP1. Nucleic Acids Res 39, 5715-5728 (2011).

26. Mooij, W., Mitsiki, E. \& Perrakis, A. ProteinCCD: enabling the design of protein truncation constructs for expression and crystallization experiments. Nucleic Acids Res (2009). doi:10.1093/nar/gkp256

27. Luna-Vargas, M. P. A. et al. Enabling high-throughput ligation-independent cloning and protein expression for the family of ubiquitin specific proteases. Journal of structural biology 175, 113-119 (2011).

28. Roosendaal, J. et al. Development, validation, and clinical application of a high-performance liquid chromatography-tandem mass spectrometry assay for the quantification of total intracellular $\beta$-decitabine nucleotides and genomic DNA incorporated $\beta$-decitabine and 5 methyl-2'-deoxycytidine. J Pharm Biomed Anal 164, 16-26 (2018).

29. Grover, R. K. et al. O-glycoside orientation is an essential aspect of base J recognition by the kinetoplastid DNA-binding protein JBP1. Angew Chem Int Ed Engl 46, 2839-2843 (2007).

30. Pernot, P. et al. Upgraded ESRF BM29 beamline for SAXS on macromolecules in solution. $J$ Synchrotron Radiat 20, 660-664 (2013).

31. Shkumatov, A. V. \& Strelkov, S. V. DATASW, a tool for HPLC-SAXS data analysis. Acta Crystallogr. D Biol. Crystallogr. 71, 1347-1350 (2015).

32. Petoukhov, M. V. et al. New developments in the ATSAS program package for small-angle scattering data analysis. J Appl Crystallogr 45, 342-350 (2012).

33. Rambo, R. P. \& Tainer, J. A. Accurate assessment of mass, models and resolution by smallangle scattering. Nature 496, 477-481 (2013).

34. Svergun, D. I. Determination of the regularization parameter in indirect-transform methods using perceptual criteria. J App/ Crystallogr 25, 495-503 (1992).

35. Rambo, R. P. \& Tainer, J. A. Characterizing flexible and intrinsically unstructured biological macromolecules by SAS using the Porod-Debye law. Biopolymers 95, 559-571 (2011).

36. Petoukhov, M. V. \& Svergun, D. I. Ambiguity assessment of small-angle scattering curves from monodisperse systems. Acta Crystallogr. D Biol. Crystallogr. 71, 1051-1058 (2015). 
37. Konarev, P. V. \& Svergun, D. I. A posteriori determination of the useful data range for smallangle scattering experiments on dilute monodisperse systems. IUCrJ 2, 352-360 (2015).

38. Svergun, D. I. Restoring Low Resolution Structure of Biological Macromolecules from Solution Scattering Using Simulated Annealing. Biophysical Journal 76, 2879-2886 (1999).

39. Kozin, M. B., Svergun, D. I.IUCr. Automated matching of high- and low-resolution structural models. J Appl Crystallogr 34, 33-41 (2001).

40. Rambo, R. P. Resolving Individual Components in Protein-RNA Complexes Using Small-Angle X-ray Scattering Experiments. Meth Enzymol 558, 363-390 (2015).

41. Kozin, M. B. \& Svergun, D. I. Automated matching of high- and low-resolution structural models. J. Appl. Cryst (2001). 34, 33-41 [doi:10.1107/S0021889800014126] 34, 1-9 (2001).

42. Svergun, D., Barberato, C. \& Koch, M. H. J. CRYSOL - a Program to Evaluate X-ray Solution Scattering of Biological Macromolecules from Atomic Coordinates. J. Appl. Cryst (1995). 28, 768-773 [doi:10.1107/S0021889895007047] 28, 1-6 (1995).

43. Holm, L. \& Laakso, L. M. Dali server update. Nucleic Acids Res 44, W351-5 (2016).

44. Heidebrecht, T. et al. The structural basis for recognition of base J containing DNA by a novel DNA binding domain in JBP1. Nucleic Acids Res 39, 5715-5728 (2011).

45. Wang, X. et al. Structural basis of Cas3 inhibition by the bacteriophage protein AcrF3. Nat Struct Mol Biol 23, 868-870 (2016).

46. Cliffe, L. J. et al. JBP1 and JBP2 proteins are Fe2+/2-oxoglutarate-dependent dioxygenases regulating hydroxylation of thymidine residues in trypanosome DNA. Journal of Biological Chemistry 287, 19886-19895 (2012).

47. Cliffe, L. J. et al. JBP1 and JBP2 are two distinct thymidine hydroxylases involved in J biosynthesis in genomic DNA of African trypanosomes. Nucleic Acids Res 37, 1452-1462 (2009).

48. Shen, A., Higgins, D. E. \& Panne, D. Recognition of AT-rich DNA binding sites by the MogR repressor. Structure/Folding and Design 17, 769-777 (2009).

49. Mohanty, B., Helder, S., Silva, A. P. G., Mackay, J. P. \& Ryan, D. P. The Chromatin Remodelling Protein CHD1 Contains a Previously Unrecognised C-Terminal Helical Domain. Journal of molecular biology 428, 4298-4314 (2016).

50. Chowdhury, S. et al. Structure Reveals Mechanisms of Viral Suppressors that Intercept a CRISPR RNA-Guided Surveillance Complex. Cell 169, 47-57.e11 (2017).

51. Dong, D. et al. Structural basis of CRISPR-SpyCas9 inhibition by an anti-CRISPR protein. Nature 546, 436-439 (2017). 
TABLES AND FIGURES LEGENDS

Table 1.

Model independent parameters for all samples used in this study.

\begin{tabular}{|l|c|c|c|c|c|c|}
\hline & $\mathrm{R}_{\text {g-Guinier }}(\AA)$ & $\mathrm{R}_{\text {g-reciprocal }}(\AA)$ & $\mathrm{D}_{\max }(\AA)$ & $\mathrm{MW}_{\text {theory }}(\mathrm{D})$ & $\mathrm{V}_{\text {Porod }}$ & $\mathrm{V}_{\mathrm{c}}$ \\
\hline Monomers & 34.3 & 34.3 & 120 & 93403 & 98.7 & 610.1 \\
\hline JBP1 & 30.6 & 30.6 & 99 & 72118 & 76.4 & 505.5 \\
JDBD & 24.8 & 24.8 & 71 & 21285 & 28.3 & 260.9 \\
J-23-DNA & 22.5 & 22.5 & 73 & 14241 & 16.2 & 201.7 \\
J-15-DNA & 15.8 & 15.8 & 51 & 9301 & 10.3 & 131.8 \\
\hline Complexes & 40.98 & 40.98 & 141 & 107644 & 107.1 & 685.8 \\
\hline JBP1:J-23-DNA & 35.6 & 36.29 & 120 & 102704 & 104 & 641.9 \\
JBP1:J-15-DNA & 21.7 & 21.67 & 70 & 35526 & 34.73 & 241.1 \\
JDBD:J-23-DNA & 21.7 &
\end{tabular}

Table 2.

Details for the phases used in MONSA for modeling, and all resulting clusters, for all complexes and sub-complexes used in this study.

\begin{tabular}{|c|c|c|c|c|c|c|c|}
\hline Complex & Phase 1 & $\begin{array}{l}\text { Phase } 2 \\
\end{array}$ & Cluster-1 & Cluster-2 & Cluster-3 & Cluster-4 & Cluster-5 \\
\hline JBP1 & $\triangle$-JDBD & JDBD & 7 & 3 & 2 & 2 & 2 \\
\hline JBP1:23-J-DNA (I) & JBP1 & 23-J-DNA & 7 & 5 & 4 & 3 & - \\
\hline JBP1:23-J-DNA (II) & $\triangle-J D B D$ & JDBD:23-J-DNA & 10 & 6 & 2 & - & - \\
\hline JBP1:15JDNA & JBP1 & 15-J-DNA & 14 & 3 & 2 & - & - \\
\hline
\end{tabular}


A

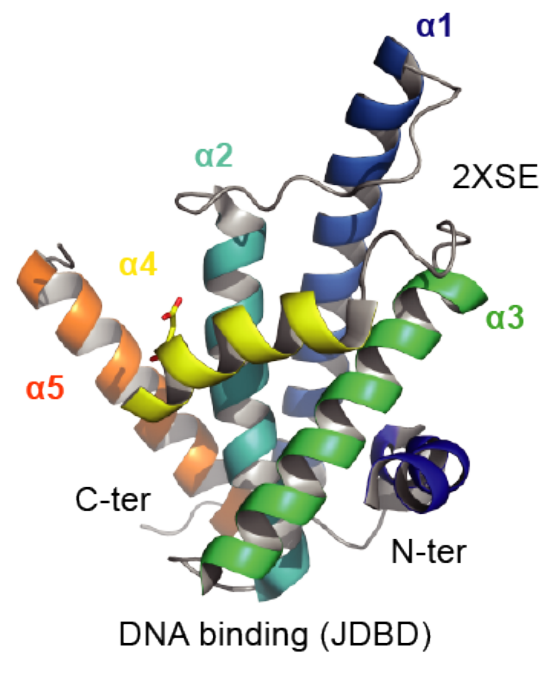

B
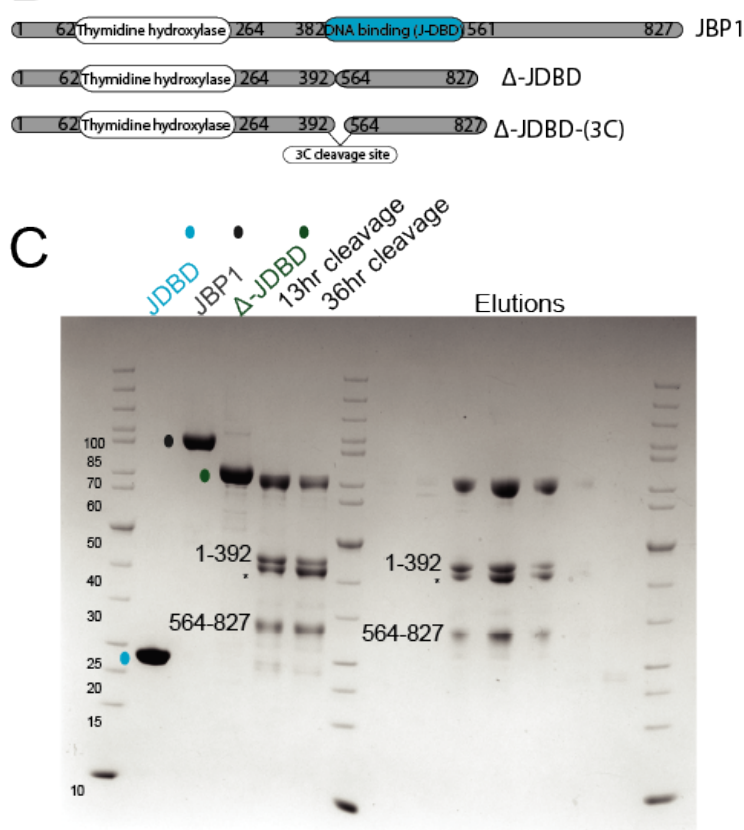

$\left.\mathrm{D}^{1500}\right]$ - JBP1

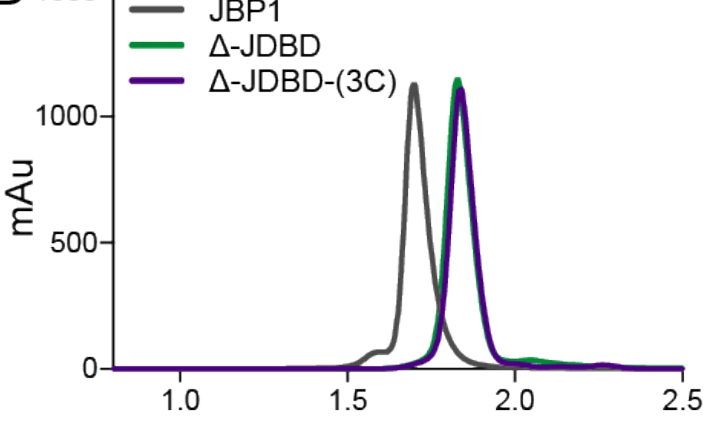

Retention Volume from SEC $(\mathrm{mL})$
Figure 1: Domain organization of JBP1. (A) cartoon representation of JDBD crystal structure, showing the characteristic helical bouquet fold containing the helix-turn-helix motif. Asp-525 is shown as stick. (B) Constructs used in this study; full-length JBP1, $\triangle$-JDBD-3C, $\triangle$-JDBD. (C) SDS-PAGE showing the overexpressed constructs in the following order: Marker, JDBD, JBP1, $\triangle$-JDBD-3C, $\Delta^{-}$ JDBD-3C products after 13 and $36 \mathrm{hr}$ incubation with $3 C$ protease, marker, SEC elution fractions of $\triangle$-JDBD-3C, after incubation with $3 \mathrm{C}$ protease. Chromatogram of the SEC for JBP1, $\triangle$-JBP1 and $\triangle$-JDBD-3C; $\triangle$-JBP1 before and after cleavage with $3 C$ protease are identical. The $\mathrm{N}$ - and C-terminal regions of $\triangle$-JDBD-3C, elute in the same peak (see panel (C) above, suggesting that there is a strong interaction between them. 


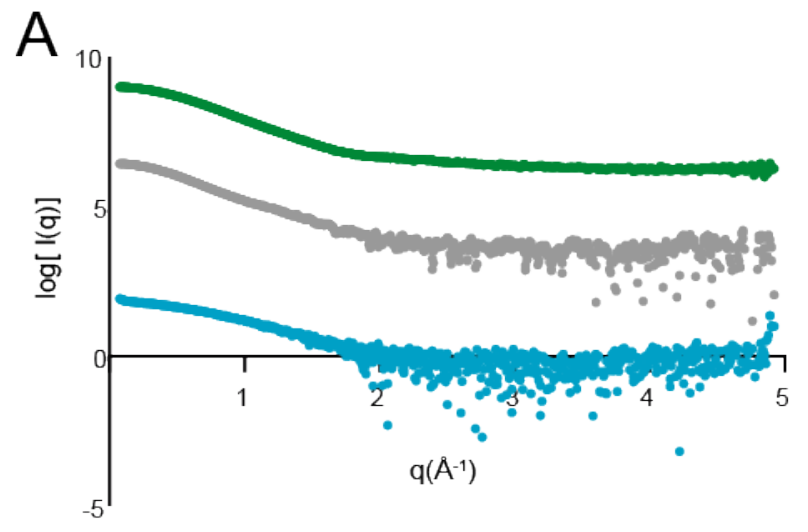

B Dimensionless Kratky
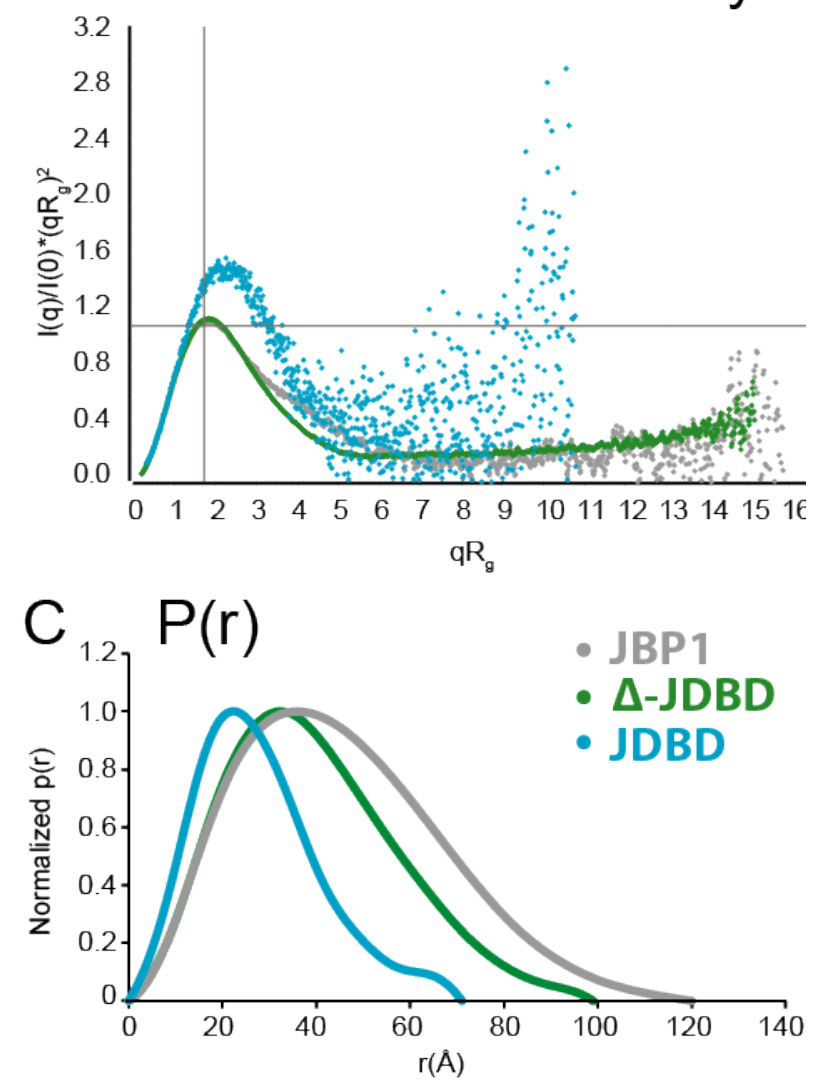

Figure 2: Folding of JBP1, $\triangle$-JDBD, JDBD and 23 J-DNA. (A) Experimental scattering curves of all components. (B) Dimensionless Kratky plot; the 1.104 maximum for an ideal compacted molecule is shown as the intersection between the 2 grey lines; (C) normalized pair distribution function for all components. 


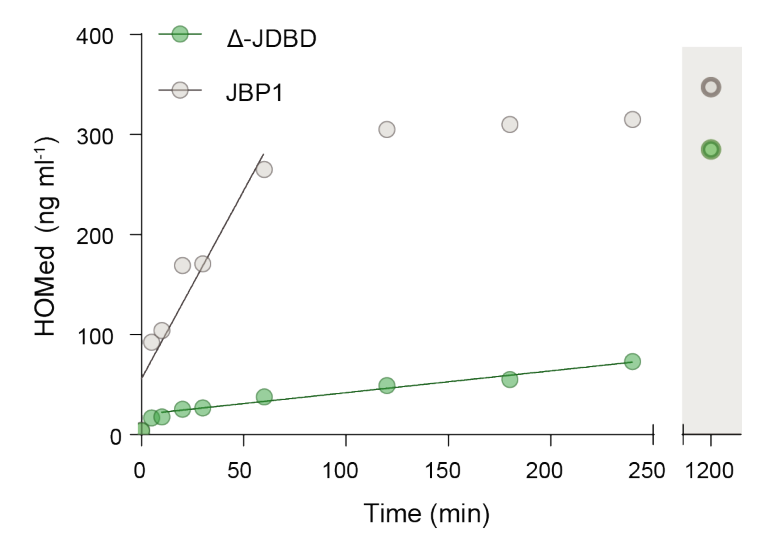

Figure 3: The apparent catalytic rates of JBP1 and $\triangle$-JDBD. The linear part of the reaction curve (5-60 min for JBP1, 10-240 minutes for $\triangle$-JDBD) are fitted with a line to estimate the rates.
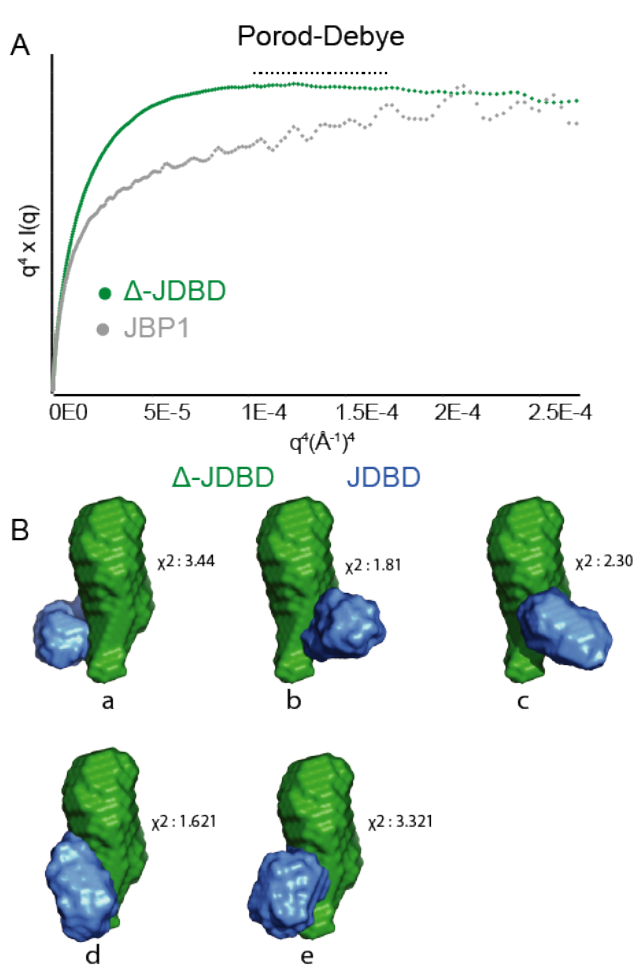

C

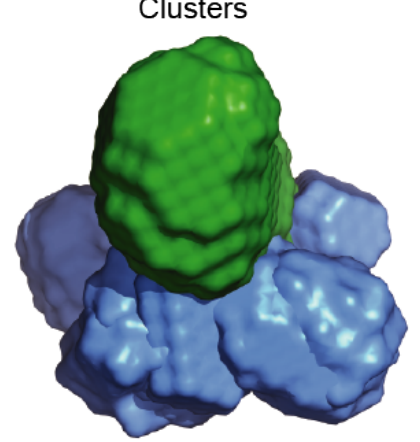

Figure 4: MONSA modelling of JBP1. (A)

Porod-Debye plot demonstrating a loss of the plateau when JDBD is present, suggesting that JBP1 is more flexible. (B) Clustered MONSA models of JBP1; $\triangle$-JDBD and JDBD are shown in green and blue, respectively; a total five clusters were identified and the fitting to the experimental data of the calculated intensities for each model is shown based on $\chi^{2}$. (C) A representation of all possible JDBD orientations shown along the long axis of $\Delta^{-}$ JDBD. Figures were prepared using the program ScÅtter and Pymol. 


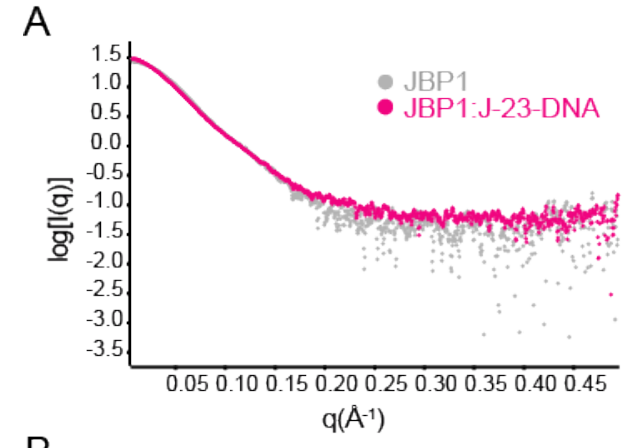

B

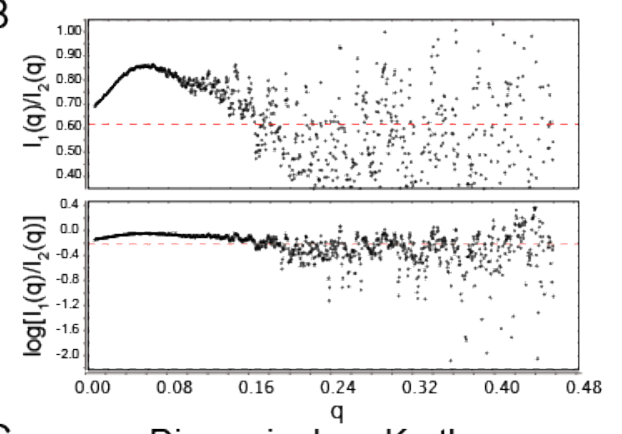

C

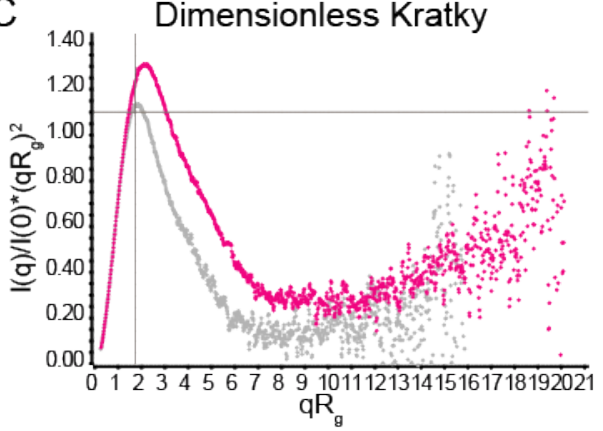

Figure 5: Comparison of JBP1, in presence and absence of J-23-DNA. (A) Experimental scattering curves of JBP1 and JBP1:J-23-DNA.

(B) Ratio plots of the two datasets. (C) Dimensionless Kratky plot for both datasets.

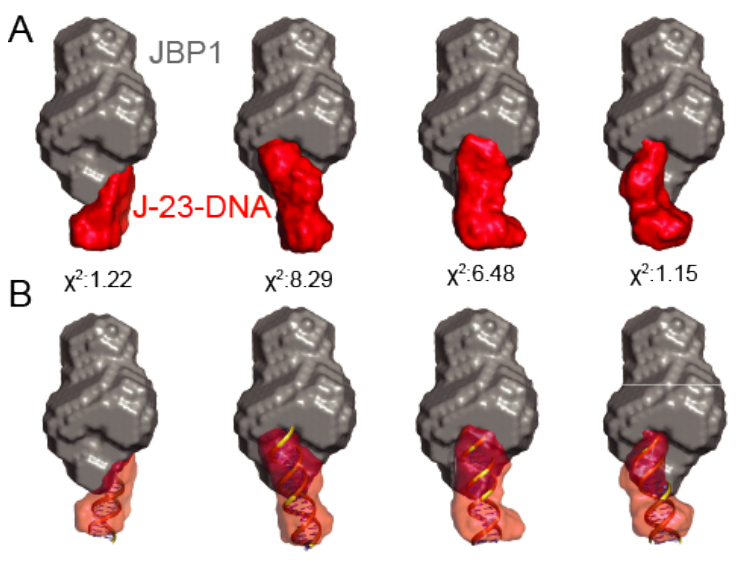

Figure 6: Model of JBP1:J-23-DNA. (A) Clusters of all JBP1:J-23-DNA MONSA models, with the $\chi^{2}$ fitting of the calculated intensities to the experimental data. (B) The J-23-DNA is superimposed on the molecular envelope generated by MONSA. Superposition was performed using SUPCOMB from the ATSAS suite. Figures generated using Pymol. 


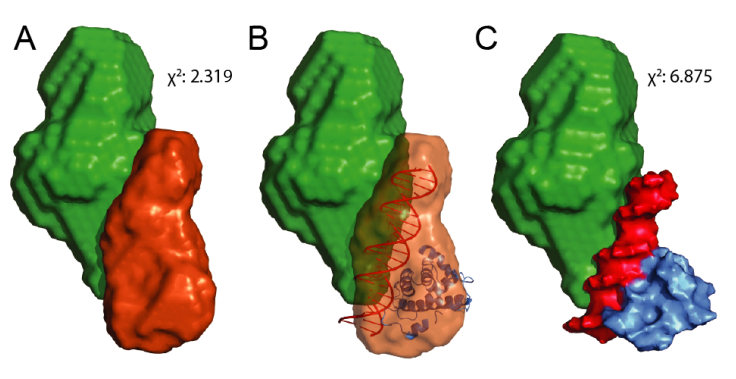

Figure 7: Model of JBP1 in presence of JDNA.

(A) Clustered MONSA model showing the relative positions of $\triangle$-JDBD and JDBD:J-23DNA sub-complex. (B) A pseudo-atomic model of JDBD:J-23-DNA fitted in the molecular envelope of the generated model. (C) Surface representation of all components. Figures were made using Pymol.

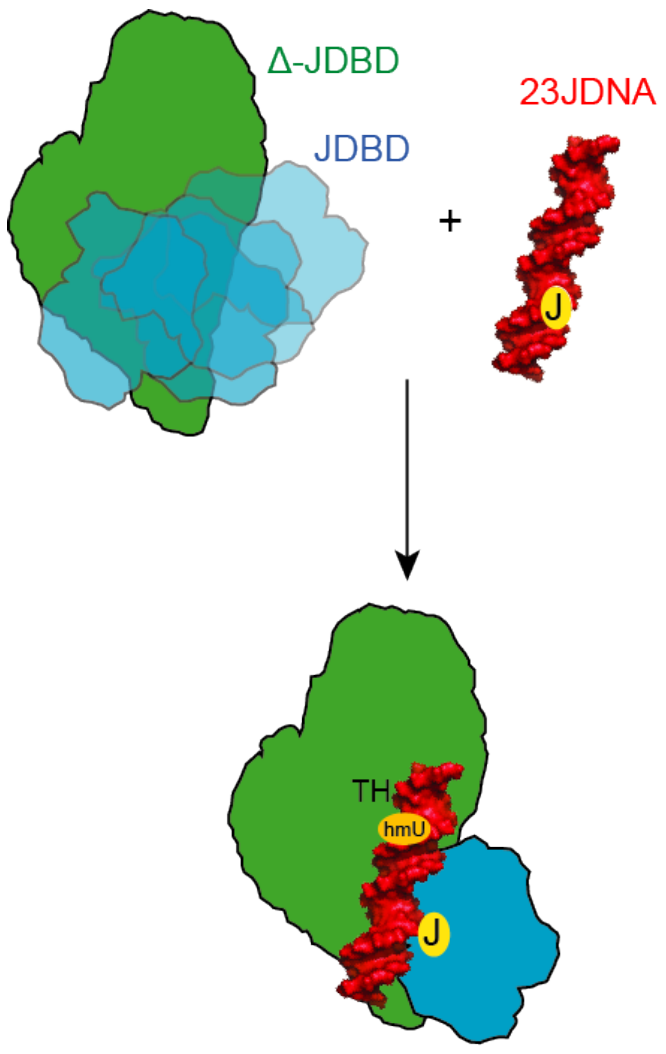

Figure 8: A model suggesting the mechanism for the recognition and maintenance of base-J. JDBD recognizes and binds base-J with high affinity, result in a conformational change that could stabilize the JDBD domain. Upon binding, JDNA is an orientation that allows the $\mathrm{TH}$-domain to be in close proximity to the thymine base that is located $13 \mathrm{bp}$ downstream on the complementary strand to promote its hydroxylation, and therefore maintenance of $J$ at specific positions in the genome of kinetoplastids. 

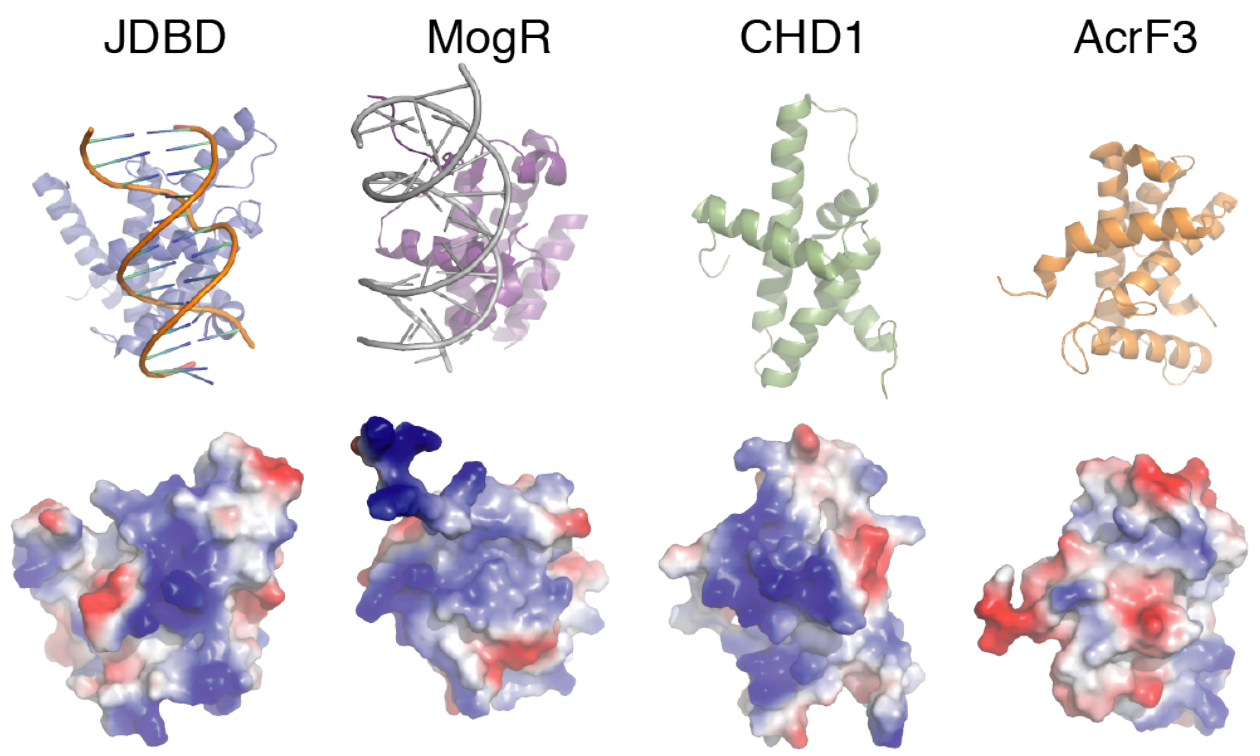

Figure 9: Cartoon models of the JDBD and homologues in the PDB (top) and the corresponding surface representations coloured by electrostatic potential. The positive blue patches are characteristic for the interaction with DNA as shown in the SAXS-based model of JDBD and the MogR crystal structure; this positive patch is entirely missing in the AcrF3 protein. 


\section{METHODS}

\section{Cloning of $\Delta$-JDBD JBP1 and $\Delta-J D B D-3 C$ JBP1}

A JBP1 synthetic gene encoding the sequence for Leishmania tarentola ${ }^{25}$ was used as the template for all constructs in this study. Primers to delete the JDBD domain and to replace it with the $3 C$ protease DNA sequence were designed using the ProteinCCD software ${ }^{26}$. To create the $\triangle$-JDBD construct, the JDBD domain was deleted using mutagenesis PCR and primers Del fW 5'- CTC GTC TGG GTG GTT TCT CTG AAA CCT CTC ACG AAA AAC GTG CTA ACT GGC TG -3' and Del_rev 5'- CAG CCA GTT AGC ACG TTT TTC GTG AGA GGT TTC AGA GAA ACC ACC CAG ACG AG -3'. The generated construct contained the $\mathrm{N}$-terminal part from residues 1-392 followed by the C-terminal part residues 564-827 without any connecting linker in between. To generate the $\triangle$-JDBD-3C construct a 3 C protease cleavage site was introduced using primers Del-DB-3C_fw 5'-CTC GTC TGG GTG GTT TCT CTG AAA CCC TGG AAG TGC TGT TTC AGG GCC CGT CTC ACG AAA -3' and Del-DB-3C_rev 5'-CAG TTA GCA CGT TTT TCG TGA GAC GGG CCC TGA AAC AGC ACT TCC AGG GTT TCA GAG AAA -3' respectively (highlighted is the DNA sequence for the 3C-protease cleavage site).

\section{Expression and purification of recombinant proteins}

All constructs of JBP1, JDBD and $\triangle-J D B D$ were inserted in the NKI-LIC-1.1 vector ${ }^{27}$ and produced as soluble proteins in E.coli. BL21 (DE3) T1R cells were used for protein overexpression. Protein production was induced with IPTG at $15^{\circ} \mathrm{C}$ for $16-18 \mathrm{hr}$. Cell lysis was performed in buffer A (20 mM Hepes/NaOH pH 7.5, $350 \mathrm{mM} \mathrm{NaCl}, 1 \mathrm{mM}$ tris(2-carboxyethyl)phosphine (TCEP)), containing $10 \mathrm{mM}$ imidazole. The lysate was bound to $\mathrm{Ni}$-chelating sepharose beads in batch mode, and elution was performed in buffer A containing $400 \mathrm{mM}$ imidazole. Affinity tags were removed by $3 \mathrm{C}$ protease cleavage overnight at $4^{\circ} \mathrm{C}$ and applied to a S75 $16 / 60$ gel filtration column.

\section{In vitro hydroxylation assays}

The thymidine hydroxylase activity assay was carried out in a reaction buffer containing: $50 \mathrm{mM}$ HEPES/ $\mathrm{NaOH}$ (pH7.6), $50 \mathrm{mM} \mathrm{NaCl}, 8 \mathrm{mM}$ ascorbic acid, $4 \mathrm{mM}$ 2-oxoglutarate, $1 \mathrm{mM} \mathrm{Fe}_{2} \mathrm{SO}_{4}, 1 \mathrm{mM}$ ADP, $20 \mu \mathrm{g} \mathrm{mL}^{-1}$ BSA and $0.5 \mathrm{mM}$ DTT. The buffer was made anaerobic by degassing it with Argon for 1h at $4^{\circ} \mathrm{C}$. The reaction was carried out at $37^{\circ} \mathrm{C}$ in a total volume of $50 \mu \mathrm{l}$, including $4 \mu \mathrm{M}$ of the protein and $15 \mu \mathrm{M}$ of 14-mer double-stranded DNA (CAGCAGCTGCAACA). Upon completion of the reaction at indicated time points, samples were stored at $-20^{\circ} \mathrm{C}$ for further prcessing.

\section{Sample preparation for mass spectrometry}

Aliquots of $20 \mu \mathrm{L}$ of the reaction mixtures were placed in $1.5 \mathrm{~mL}$ reaction tubes, and incubated at $95^{\circ} \mathrm{C}$ for 3 minutes followed by rapid cooling on ice, to denature the double stranded DNA to single 
stranded oligonucleotides. In each tube, 4 units of Nuclease P1 (Sigma-Aldrich, St. Louis, MO)) were added together with $100 \mu \mathrm{L}$ digest buffer, containing $0.04 \mathrm{mM}$ DFAM, $3.25 \mathrm{mM}$ ammonium acetate $\mathrm{pH} 5.0$ and $0.5 \mathrm{mM}$ zinc chloride). The samples were incubated at $65^{\circ} \mathrm{C}$ for 10 minutes to convert the oligonucleotides into single nucleotides. We then added $20 \mu \mathrm{L}$ of Trizma base $\mathrm{pH} 8.5$ and 4 units of alkaline phosphatase (Roche Life Science, Indianapolis, IN) and vigorously mixed for approximately 10 seconds. Samples were incubated at $37{ }^{\circ} \mathrm{C}$ (heating block) for 1 hour to allow for nucleotide to nucleoside conversion, after which we added $20 \mu \mathrm{L}$ of $300 \mathrm{mM}$ ammonium acetate $\mathrm{pH} 5.0$ and evaporate to dryness at $40^{\circ} \mathrm{C}$ in a TurboVap LV (Biotage, Uppsala, Sweden). Finally, we added $50 \mu \mathrm{L}$ of $5 \mathrm{mM}$ ammonium acetate in water - acetonitrile $(2: 98, \mathrm{v} / \mathrm{v})$ and vigorously mixed for approximately $1 \mathrm{~min}$.

\section{Measurement of hmU by mass spectrometry}

Oligonucleotide $\mathrm{HmU}$ content was analysed as the released amount of 5'-hydroxymethyl-2'deoxyuridine (HOMedU) after sample processing. A reference standard of HOMedU (Santa Cruz Biotechnology, Inc., Dallas, TX) was used to prepare calibration standards for HOMedU sample quantification.

For quantification, the HPLC-MS/MS system consisted of a QTRAP 5500 tandem mass spectrometer (Sciex, Framingham, MA, USA) coupled to an HPLC Acquity I Class pump (Waters, Milford, MA, USA). The HPLC system was equipped with a FTN I-Class autosampler and I-Class column oven (Waters). Data acquisition was performed using Analyst 1.6.2. software (Sciex).

The HPLC-MS/MS system was based on a previously developed method to quantify decitabine DNA incorporation ${ }^{28}$. This assay was modified to allow for HOMedU quantification in the positive electrospray ionization mode by using the following mass-to-charge ratio $(\mathrm{m} / \mathrm{z})$ transition: $257.0 \rightarrow$ 124.0. The remaining settings of the method were unchanged.

\section{Preparation of J-DNA and JDBD:J-DNA and JBP1:J-DNA complex}

J-DNA oligos ${ }^{29}$ were mixed with their complementary strand and annealed as previously described ${ }^{18}$. Briefly, the hmC-containing oligonucleotide and the complementary strand were dissolved in water to a concentration of $100 \mu \mathrm{M}$ and then heat-annealed. The double-stranded oligonucleotide was then glucosylated by the T4 Phage $\beta$-glucosyltransferase (T4-BGT) from New England BioLabs according to manufacturer's instructions. To create the protein: J-DNA complexes, $1 \mathrm{mg} \mathrm{ml}^{-1}$ of JBP1 was mixed with J-DNA at 1:1.1 molar ratio and then concentrated with Amicon concentrators. The same procedure was used for making the JDBD:J-DNA sub-complexes. The sequences used in this study were 23-J-DNA TCGATTJGTTCATAGACTAATAC and J-15-DNA TAGAACCCJAACCAT. 
SEC-SAXS data collection and analysis

Synchrotron X-ray data for all components were collected on a Pilatus $1 \mathrm{M}$ detector at the ESRF beamline $B M 29^{30}$. About $40 \mu \mathrm{l}$ of each sample, at a concentration 3-10 $\mathrm{mg} \mathrm{ml}^{-1}$ were loaded onto a Superose-6 column (Supplementary Table 1). The flow rate for SAXS data collection was $0.2 \mathrm{ml} \mathrm{min}$ and a scattering profile was integrated every second. Frames for each dataset were selected based on the examination of the Size Exclusion profile together with the calculated $R_{g}$ and $D_{\max }$ values. At least 20 frames for each dataset were selected, scaled and averaged using PRIMUS following the standard procedures (Supplementary Table 1). For the JDBD:23-J-DNA and JBP1:J-15-DNA, frames were analyzed with DATASW ${ }^{31}$.

\section{Model-independent analysis of SAXS data}

SAXS data analysis was performed using the PRIMUS ${ }^{32}$ and ScÅtter ${ }^{33}$ software packages. The forward scattering $I(0)$ was evaluated using the Guinier approximation (8) assuming the formula $l(q)=$ I(0) $\exp \left(-\left(q R_{g}\right)^{2} / 3\right)$ for a very small range of momentum transfer values $\left(q \mathrm{R}_{\mathrm{g}}<1.3\right)$. Calculation of the pair distribution function and maximum distance $D_{\max }$ was performed using $\mathrm{GNOM}^{34}$. The $\mathrm{R}_{\mathrm{g}}$ was estimated by Guinier approximation. The molecular mass was calculated using the Porod volume, and the $Q_{R}$ method $^{33,35}$. Ambiguity of all datasets was measured with AMBIMETER ${ }^{36}$. The useful range for each dataset was determined by SHANUM analysis ${ }^{37}$ prior to proceeding to $a b$ initio modeling.

\section{$A b$ initio modeling using SAXS data}

Molecular envelopes of $a b$ initio created models, were made for all the components using $D^{D A M M I N}{ }^{38}$. Ten individual models were created for each component and averaging was performed using DAMAVER. Fitting of atomic resolution structures to molecular envelopes was performed using SUPCOMB ${ }^{39}$.

To resolve the relative positions of individual subunits of the JBP1 structure a volumetric analysis was performed using the program MONSA, an extension of the DAMMIN algorithm ${ }^{38}$, following an approach similar to the one described $\mathrm{in}^{40}$. MONSA allows $a b$ initio modeling of macromolecular complexes by fitting simultaneously multiple experimental datasets. The search volume is defined as a sphere with radius equal to half the maximum dimension $\left(D_{\max }\right)$ of the complex of study. $A$ minimization algorithm based on simulated annealing fits the experimental datasets of each component (phase), while all components together should fit to the experimental dataset of the corresponding complex. In case of a multi-component complex, each different phase is assigned a different contrast ( 1 for protein, 2 for nucleic acid, 0 for solvent). In our approach we treat as a phase either different components (protein and DNA) but also the two distinct folding units that 
make JBP1 as we establish them in this work ( $\triangle \mathrm{JDBD}$ and JDBD). At least 20 individual runs for each complex (particle) were created. The online version of MONSA was used for all models generated in this study.

In case of a two-body modeling that consists of two components $X: Y, X$ denotes the component with the larger mass, and $y$ the smaller mass. In our experiments, $X: y$ would thus be JBP1:23-J-DNA or $\Delta$ JDBD:JDBD. This modeling assumes that each component (phase) does not undergo conformational changes upon complex formation, or that the difference at that resolution is negligible.

Examination of the parameters stored in the log file, for each MONSA run show the fitting and the calculated $R_{g}$ values for each component from each individual MONSA run. To evaluate each MONSA modeling run we compared the $R_{g}$ derived from the experimental dataset for each component, with the calculated $R_{g}$ for each phase calculated for each MONSA model and stored in the log file. Runs with calculated $R_{g}$ values that differ significantly from the experimental-data derived $R_{g}$ were excluded from the analysis.

\section{Aligning models generated by MONSA}

To compare MONSA models, we wanted to visualize the different position of each phase $y$ relative to $X$, in a common reference framework. We therefore chose to first align all phases $X_{i}$ from each individual run. Then the transformation matrix of each $X_{i}$ component was used to also transform the corresponding $y_{i}$ phase. The chosen procedure highlights the possible relative positions of $y_{i}$ relative to $X_{i}$. This is preferable to aligning all complexes $X_{i}: y_{i}$ to each other, which only yields the average relative position.

For that we developed a script in Python (available as supplementary information), that performs the following operations. DAMAVER is used to align all models of $X_{i}$, the component that has the larger mass. DAMAVER superimposes all models to each other, averages them, selects the best model based on the normalized spatial discrepancy (NSD) metric as the reference model $\left(\mathrm{X}_{\text {ref }}\right)$, and aligns all models to $X_{\text {ref. }}$ DAMAVER creates a new PDB file for each $X_{i}$ in the new aligned position, also containing the transformation matrix $\left(T_{i}\right)$ that aligns each model $\left(X_{i}\right)$ with the reference $\left(X_{\text {ref }}\right)$. Then, the transformation matrix $T_{i}$ is used to transform every $y_{i}$ phase. Finally, DAMCLUST is used to cluster the transformed $y_{i}$ phases, effectively creating clusters that have the $y_{i}$ component in similar orientations. These clusters, define the conformational clusters of the to $X_{i}: y_{i}$ complex.

Each cluster is presented as the average model of all $X_{i}$ components, and the average of the $y_{i}$ components for each cluster. Singleton clusters were excluded from our analysis. To evaluate the clustered models we examined their fit to the experimental data based on $\chi^{2}$ analysis. 
To fit atomic resolution structures on molecular envelops generated by either DAMMIN or MONSA the program SUPCOMB ${ }^{41}$ was used.

To calculate the $\chi^{2}$ values of the hybrid dummy atoms model of $\Delta$-JBP1 and the atomic model of JDBD:23-J-DNA we transformed the hybrid model using CRYSOL ${ }^{42}$ and compared it to the experimental data of the complex.

\section{Structure similarity searches}

Structure similarity searches were carried out by DALI searches against the whole PDB ${ }^{43}$. DALI returned new hits compared to previous searches ${ }^{44}$. We then inspected the top hits manually. The recognition helix, the supporting helix, as well as two of the other helices and the connectivity we have described for the helical bouquet fold of JDBD ${ }^{44}$ were present in the top 1 (self), 2, 3, and 5 hits. Hit nr. 5 (Z-score 5.2, RMSD 3.0, 10\% identity over 84 aligned residues) is the motility gene receptor MogR that we have previously identified as a JDBD structural homolog. Hit nr. 2 (Z-score 6.7, RMSD 3.0, $10 \%$ identity over 97 aligned residues) is AcrF3, a protein encoded by gene 35 from phage JBD5 that has been reported to specifically inhibit the Cas3 protein of $P$. aeruginosa strain UCBPP-PA14 (PaCas3) and to counteract the type I-F CRISPR-Cas system ${ }^{45}$. Hit nr. 3 (Z-score 6.0, RMSD 4.0, 11\% identity over 94 aligned residues) is a chromodomain helicase DNA binding protein. Hit 4 has the lowest sequence identity (6\%), the recognition helix is missing, and is a potassium channel with no functional homology. Hits in position 5 and below were too distant to consider, as judged by Z-scores (4.8 and below), RMSD (4.5 and above) and had no functional similarity (DNA binding). 


\section{Supplementary Information}

\section{Supplemental Table 1}

\begin{tabular}{|c|c|c|}
\hline & Concentration $\left(\mathrm{mg} \mathrm{ml}^{-1}\right)$ & Frames \\
\hline \multicolumn{3}{|l|}{ Monomers } \\
\hline JBP1 & 8.7 & $724-758$ \\
\hline$\triangle$-JDBD & 10.3 & $757-806$ \\
\hline$J \mathrm{JBD}$ & 3.75 & $728-761$ \\
\hline 23-JDNA & 4.1 & $780-810$ \\
\hline 15-JDNA & 3.4 & $880-920$ \\
\hline \multicolumn{3}{|l|}{ Complexes } \\
\hline JBP1-23JDNA & 7.6 & 691-721 \\
\hline JBP1-15JDNA & 5.5 & $700-730$ \\
\hline JDBD-23JDNA & 4.7 & $651-671$ \\
\hline
\end{tabular}

\section{Supplementary Figure 1}

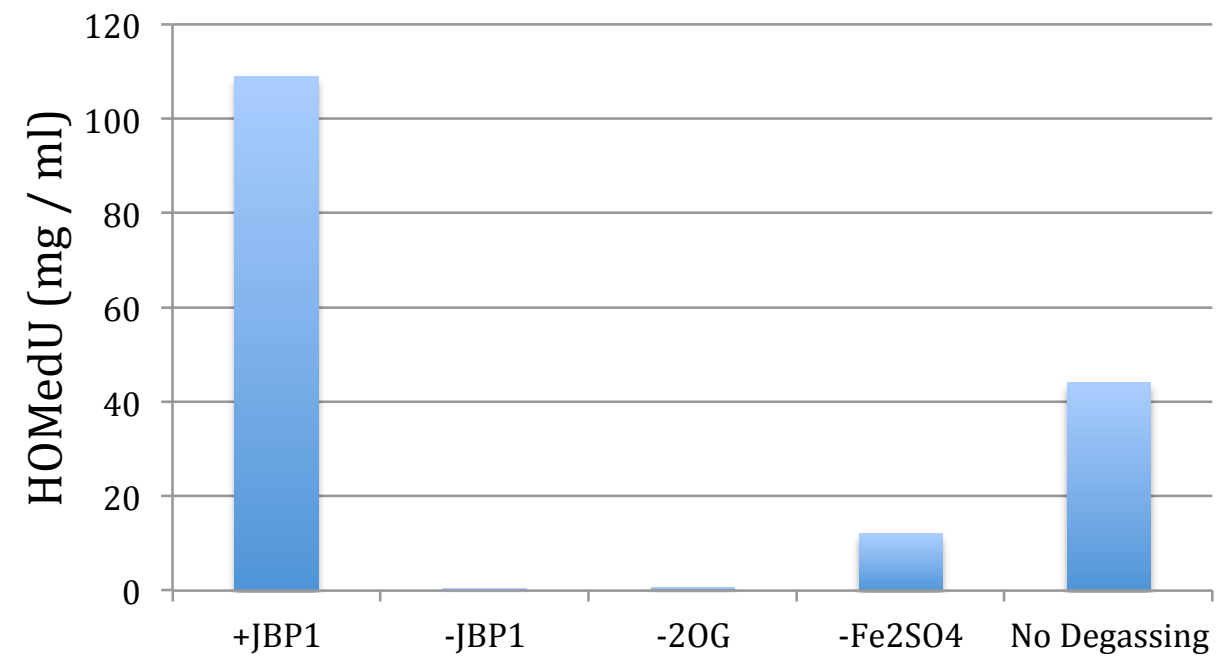

Amount of $\mathrm{hmU}$ produced by JBP1 under the conditions we describe in methods, and related controls without protein, co-factor, iron, and without degassing. 


\section{Supplementary Figure 2}

A

B
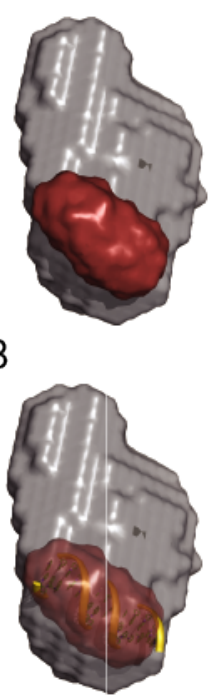

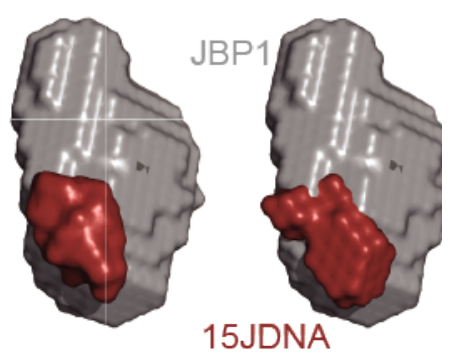

15JDNA

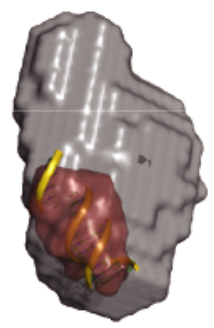

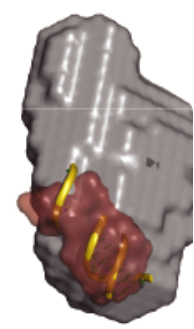

Model of JBP1:J-15-DNA. (A) Clusters of all JBP1:J-15-DNA MONSA models, with the $\chi^{2}$ fitting of the calculated intensities to the experimental data. (B) The J-15DNA is superimposed on the molecular envelope generated by MONSA. Superposition was performed using SUPCOMB from the ATSAS suite

\section{Supplementary Figure 3}

Cluster $1 ; 10$ members
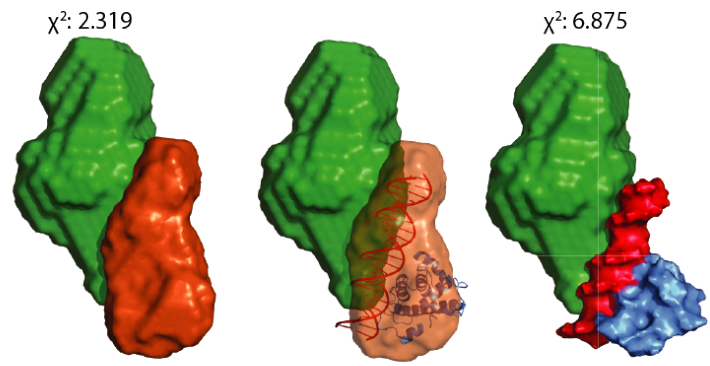

Cluster 2; 6 members
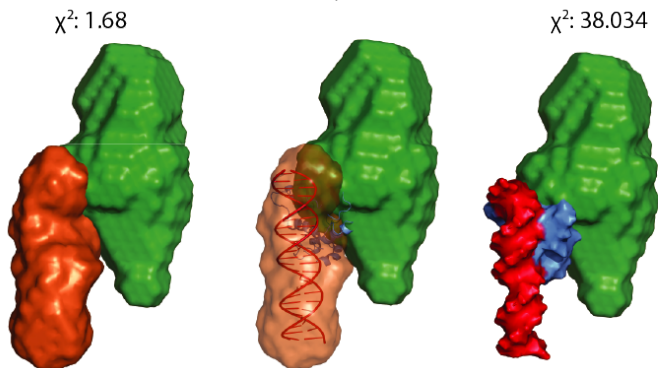

Cluster 3; 2 members

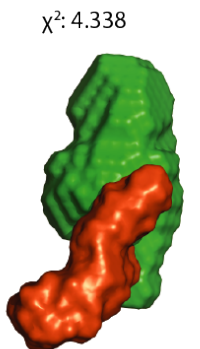

$$
X^{2}: 17.182
$$
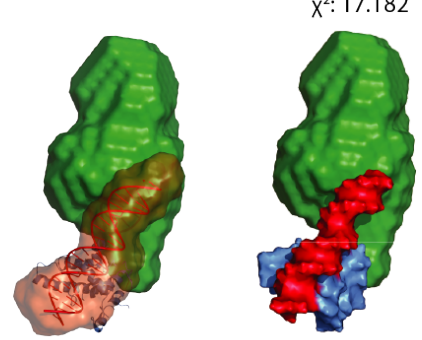

All MONSA model clusters of JBP1 in presence of JDNA. From left to right, MONSA models of the relative positions of $\triangle$-JDBD and JDBD:J23-DNA sub-complex, a pseudo-atomic model of JDBD:J-23-DNA fitted in the molecular envelope of the generated model; and a surface representation of all components. Figures were made using Pymol. 